\title{
C57BL/6 Mouse
}

National Cancer Institute

\section{Source}

National Cancer Institute. C57BL/6 Mouse. NCI Thesaurus. Code C14424.

Derived by Little (1921) from A Lathrop stocks and separated out before 1937, the C57BL/6 mouse has a black coat and is one of the most widely used inbred strains. It accounts for $14 \%$ of all mouse inbred strain laboratory usage. The strain carries a $Y$ chromosome of Asian Mus musculus orig in and a LINE-1 element from Mus spretus, suggesting that up to $6.5 \%$ of the genome is Mus spretus in origin. Substrain C57BL/6 differs from other substrains at multiple loci, including H9, Igh2 and Lv, on chromosome 4. This mouse model is prone to the development of fatty lesions in the aorta similar to atheromatous plaque in humans, as well as hyperglycemia, hyperinsulinemia, hypercholesterolemia and non-insulin-dependent diabetes mellitus in response to a high fat diet. The C57BL/6 strain exhibits good reproductive performance and hermaphroditism in this substrain has been shown to occur. 